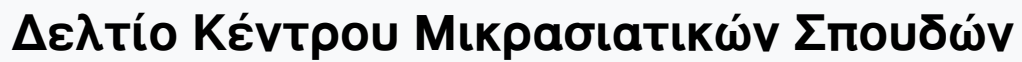

Tóp. 6 (1986)

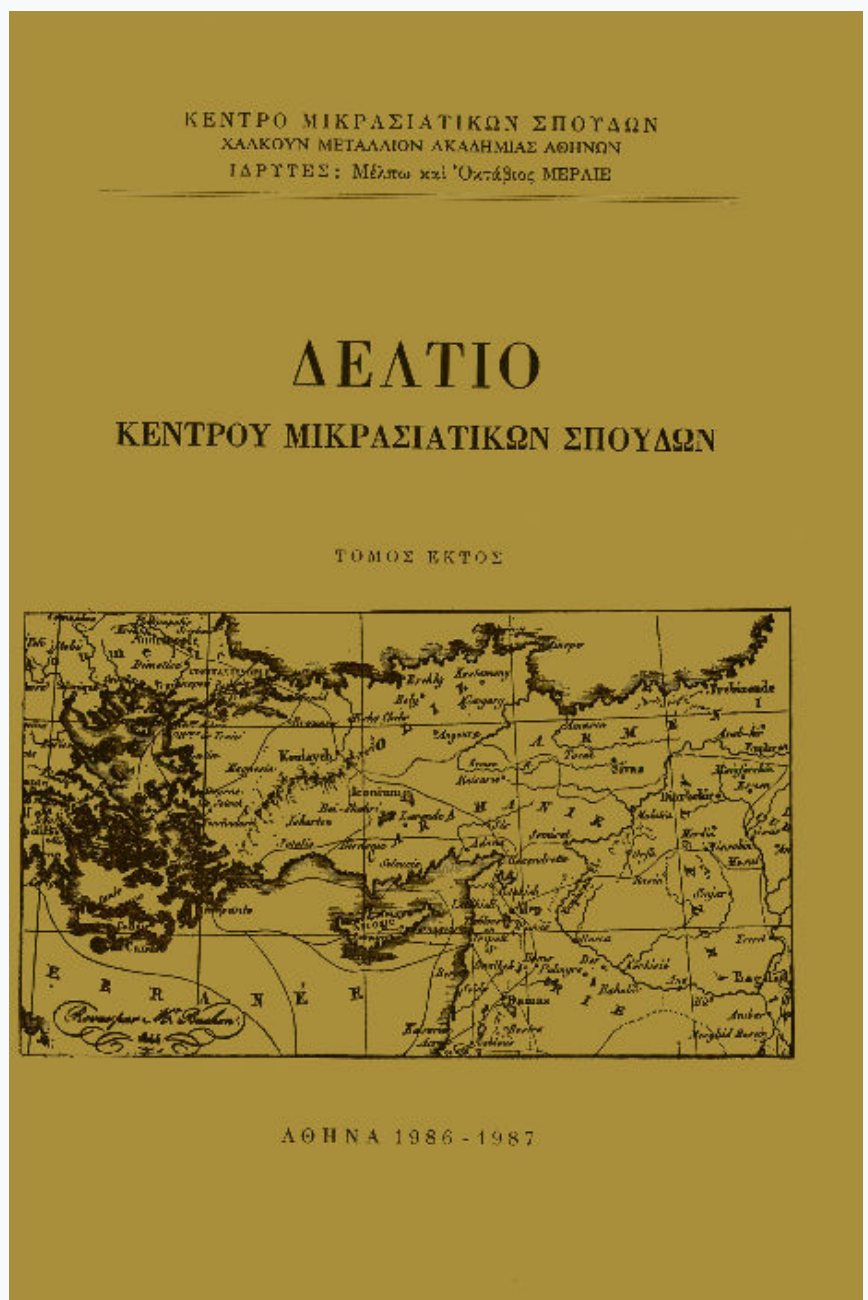

\section{Rapport de mission}

Maria Couroucli, Marie-Dominique Mouton

doi: $10.12681 /$ deltiokms.122

Copyright $\odot$ 2015, Maria Couroucli, Marie-Dominique Mouton

\section{(c) (i) (9)}

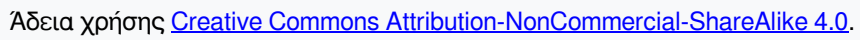

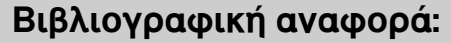

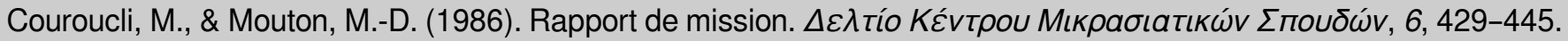
https://doi.org/10.12681/deltiokms.122 
XPONIKA 


\section{RAPPORT DE MISSION}

Le projet initial ayant motivé la mission à Athènes de Maria Couroucli, du 6/12 au 16/12, et de Marie-Dominique Mouton du 6/12 au 12/12/1987 consistait à étudier la mise au point des procédures permettant l'informatisation conjointe de deux fonds d'archives: le fonds d'ethnographie et de folklore turcs de P.N. Boratav, déposé à la bibliothèque du Laboratoire d'ethnologie et de sociologie comparative (Université de Paris X - Nanterre) et le fonds d'ethnographie et de folklore micrasiatique conservé au Centre d'Études d'Asie Mineure (Athènes).

Nous n'évoquerons pas, dans ce rapport, le fonds P.N. Boratav, nous contentant de fournir, en annexe, le plan de classement général des documents, par contre, il nous a semblé important, avant d'envisager les diverses pistes du développement d'un projet coopératif, de rendre compte de la richesse des différents fonds d'archives et d'ouvrages détenus au Centre d'Études d'Asie Mineure et de la méthodologie ayant soustendu leur constitution et leur organisation.

\section{LE CENTRE D'ÉTUDES D'ASIE MINEURE}

Le Centre d'Études d'Asie Mineure est né à Athènes, en 1930, de l'initiative de Madame Melpo Merlier, encouragée et aidée par son mari, Octave Merlier, alors directeur de l'Institut français d'Athènes.

C'est en préparant une campagne d'enregistrement des chansons populaires grecques que lui apparut la nécessité d'organiser et de systématiser les recherches folkloriques déjà entreprises à cette occasion, en particulier auprès des groupes de réfugiés d'Asie Mineure, dans le cadre du Syllogue pour l'enregistrement de la chanson populaire qu'elle avait antérieurement créé, encouragée en cela par Hubert Pernot, grand helléniste et directeur de l'Institut de Phonétique et du Musée de la Parole de l'Université de Paris. La préparation de ces enregistrements, qui eurent lieu en novembre-décembre 1930 et en novembre 1931 et permi- 


\section{XPONIKA}

rent de recueillir 573 chansons dont 256 chansons de réfugiés, avait donné lieu à un long travail, non seulement pour retrouver des membres des différentes communautés, mais également pour mieux connaître les musiciens et leur cadre de vie, spécialement lorsqu'il s'agissait de réfugiés venant de régions mal connues comme la Cappadoce.

C'est ainsi que le travail de recueil des traditions populaires des populations d'Asie Mineure devint un but en lui-même et que fut créé le Centre d'Études d'Asie Mineure. Comme le dit Octave Merlier (1974, p. 26): «...les enregistrements se poursuivirent sans cesse. Mais l'intérêt de la chanson et de la musique populaire a cédé devant celui de l'ethnographie micrasiatique».

Tel que l'on peut l'observer aujourd'hui, le Centre regroupe un certain nombre de documents: les archives musicales, les archives d'Asie Mineure auxquelles il faut adjoindre des récits et histoires de vie, oeuvres des réfugiés eux-mêmes, un fonds de cartes établies par les membres du Centre, un fonds de photographies, des documents officiels: registres ramenés d'Asie Mineure par les réfugiés et enfin une bibliothèque composée de plusieurs ensembles d'ouvrages. Est également conservée au Centre la correspondance de Melpo et d'Octave Merlier.

Nous allons successivement envisager les différents ensembles: archives musicales, archives d'Asie Mineure et bibliothèque en essayant d'analyser leur composition et de définir les principes ayant servis à leur organisation.

\section{A. Les archives musicales}

Primitivement les archives musicales de folklore se composaient de 222 disques, résultat des enregistrements de 1930-31: 204,5 disques consacrés aux chansons et 17,5 à la musique sacrée, ce qui correspond aux 573 chansons et 66 chants d'Église recueillis.

Pour chacune des chansons, une fiche permet de connaître:

- l'origine géographique du chanteur (pour les réfugiés, le lieu d'origine avant leur installation en Grèce): Pays, département (nome), province (éparchie), village.

- le genre du chant: chanson cleftiqùe, acritique, mirologue, chanson d'amour, etc. Le genre est défini en référence à la classification de Nicolas Politis, créateur des Archives littéraires de folklore, et non d'après l'avis du chanteur.

- la langue

- la première phrase de la chanson, puisque de façon courante la plupart 


\section{XPONIKA}

des chants du folklore ne possèdent pas de titre mais sont désignés par leurs premiers mots (dans le cas où l'on possède un titre, le titre et la première phrase sont indiqués).

- les premières notes de la ligne musicale

- le(s) nom(s) du (ou des) chanteur(s), du (ou des) musicien(s)

- la présence ou non d'instruments de musique accompagnant le chanteur

- les références de l'enregistrement: lieu, date, responsable

- la bibliographie: recueils musicaux de chansons populaires grecques si la chanson y figure, travaux d'analyse et de critique concernant la chanson.

Il existe également un fichier, par nom de chanteur. Sur chaque fiche figurent la photographie du chanteur, des indications biographiques ainsi que quelques renseignements sur la personnalité du chanteur et ses capacités d'informateur.

Notons qu'à cette période un certain nombre de recueils musicaux a été dépouillé et les chansons y figurant mises en fiches systématiquement.

Par la suite, les enregistrements exécutés pour le Centre ou donnés au Centre, ont été traités sensiblement de manière identique. Un nombre d'enregistrements relativement important reste, par ailleurs, à dépouiller.

\section{B. Les archives d'ethnographie micrasiatique}

Ces archives sont donc constituées des informations recueillies auprès des Grecs réfugiés d'Asie Mineure. Interrogés par des membres du Centre, plus ou moins bien formés à l'ethnographie et au travail de folkloriste, mais disposant dans tous les cas d'un questionnaire très précis pour guider leurs enquêtes. Les réfugiés évoquent à travers un certain nombre de questions, leur village, leur mode de vie en Asie Mineure ainsi que les conditions de leur rapatriement en 1922. Les interviews cessent en 1970.

Telles qu'elles se présentent aujourd'hui, les archives sont organisées selon un classement géographique. En effet, pour mieux resituer les informations recueillies, Melpo Merlier et son équipe avaient décidé de reconstituer les divisions géographiques de l'Asie Mineure hellénique, redonnant aux provinces leurs noms antiques (Bithynie, Pont, Cappadoce...), et de créer des régions constituées à partir des centres urbains importants et des villages grecs et mixtes liés à eux par la proximité spatiale, les relations économiques et administratives.

Pour chaque village (que l'on trouve donc classé matériellement à 
l'intérieur de la région et de la province), on trouve des fiches organisées selon trois axes principaux: la géographie, physique et humaine, la vie sociale (vie quotidienne, vie religieuse, sciences...) et l'histoire. On en trouvera en annexe le plan détaillé.

Sur chaque fiche sont indiqués très nettement le nom du chercheur ayant recueilli les informations, la date de l'enquête, le nom du village et le nom de l'informateur.

Il faut noter que la part la plus importante de ces documents concerne la Cappadoce, choisie en fonction du peu de travaux menés sur l'hellénisme de cette région et qui se trouve également, du fait de son plus grand isolement, la province ayant le moins subi d'influences culturelles. En chiffre cela donne:

42454 pages de matériel oral sur la Cappadoce

138815 pages au total (cf O. Merlier, 1974, p. 40, 41)

\section{Travaux manuscrits:}

Ces archives sont également constituées de travaux manuscrits (tapés à la machine par les soins du Centre), histoires de vie, souvenirs sur divers sujets (médecine populaire, légendes...), rédigés par les réfugiés d'Asie Mineure. 35 de ces documents portent sur la Cappadoce. Les travaux les plus anciens ont fait l'objet d'un catalogue.

\section{Cartothèque:}

Un fonds cartographique a été constitué. Il est composé de 85 cartes destinées à situer et éventuellement à vérifier les informations recueillies auprès des réfugiés. Un certain nombre de croquis, dont une bonne part exécutée par les informateurs eux-mêmes à l'appui de leur récit (objets usuels, costumes, sites naturels) est également conservé.

\section{Photothèque:}

Une photothèque est composée, pour la part la plus importante, de photographies prises en Cappadoce dans les années 50, ainsi que de photographies des informateurs, en particulier des musiciens et de leurs instruments.

\section{Documents officiels:}

Il ne faut pas oublier enfin, les documents officiels, registres paroissiaux, principalement, emportés par les réfugiés lors de leur exode. 34 sont déposés au Centre. 


\section{La Bibliothèque}

Plusieurs ensembles la composent, que l'on peut répartir en un fonds général et trois fonds spécifiques.

\section{Fonds général:}

Il est lui-même divisé en plusieurs ensembles d'ouvrages. Le fonds le plus important en nombre et sans doute le plus ancien, est organisé selon une classification géographique, identique à celle utilisée pour les archives elles-mêmes. On trouve également des ouvrages généraux portant sur l'histoire et classés chronologiquement selon la période de l'histoire dont ils traitent: avant 1919, 1919-1922, après 1922, des brochures et des tirés-à-part organisés selon l'ordre alphabétique d'auteur, des périodiques de philologie, d'histoire et de folklore, émanant la plupart du temps de sociétés savantes créées par les différents groupes de réfugiés, une collection d'un journal grec d'Istanbul (1861-1922), des dictionnaires et une belle collection d'almanachs anciens.

A ce fonds général on peut également adjoindre une ensemble d'ouvrages sur l'histoire ottomane et la période kémaliste (anglais, français, allemands) un ensemble d'ouvrages sur la religion grecque orthodoxe et sur le folklore, enfin un fonds de périodiques composé en particulier de publications provenant des pays slaves et des Balkans.

\section{Fonds turc:}

Il est composé d'ouvrages récents, classés par thème, de bibliographies et de collections de périodiques dont Belleten (Türk Tarih Kumuru) publié à Ankara depuis 1937.

\section{Fonds de textes karamanli:}

Ces ouvrages (rédigés en langue turque, mais imprimés avec des caractères grecs) sont classés selon l'ordre chronologique de leur date de publication.

\section{Bibliothèque d'un lettré de Smyrne:}

Acquisition la plus récente du Centre, cette bibliothèque, autrefois possession d'un lettré de Smyrne, se compose d'ouvrages uniquement édités à Smyrne, en grec. Ils portent sur des sujets variés et sont classés par ordre chronologique d'édition.

Un catalogue sur fiche a été établi pour tout ou partie du fonds général. 


\section{PROJET D'INFORMATISATION CONJOINTE}

Telles que nous venons de les décrire, l'importance numérique des fonds conservés au Centre et leur richesse laissent présager des difficultés multiples que poserait une informatisation. Néanmoins, une telle opération faciliterait grandement la diffusion de l'information sur les documents détenus au Centre vers d'autres institutions en Grèce et à l'étranger, et surtout peut-être elle permettrait une exploitation scientifique rendant possible une recherche de type comparatif.

Si l'on devait envisager une informatisation des archives, elle devrait être étudiée de manière à englober, à terme, les différents documents: archives de musique aussi bien qu'archives d'ethnographie et de folklore, cartes, photographies, histoires de vie, documents officiels et probablement aussi la correspondance d'Octave et Melpo Merlier qui apporterait beaucoup à la connaissance de l'histoire de l'ethnographie grecque et au philhellénisme des années qui ont suivi les évènements tragiques d'Asie Mineure.

Il semble, à l'heure actuelle, que les responsables du Centre envisagent d'entreprendre un plan d'informatisation en commençant par les différents fonds de la bibliothèque. Pour notre part, nous souhaitons vivement que les choix effectués pour la réalisation de ce projet permettent technıquement l'échange ultérieur de données. Il nous paraîtrait spécialement utile également de prévoir un travail commun de mise au point d'un vocabulaire contrôlé qui puisse servir à indexer, dans un premier temps les ouvrages de la bibliothèque du Centre et ultérieurement d' autres composantes du fonds et que nous utiliserions, au Laboratoire d' ethnologie, pour affiner notre indexation des ouvrages portant sur la Grèce et l'Asie Mineure hellénique ainsi que pour pratiquer notre indexation du fonds Boratav. Ce vocabulaire contrôlé devrait bien entendu être bilingue.

Un autre travail préparatoire devrait consister en la saisie d'un index informatisé recensant à la fois les noms géographiques helléniques utilisés dans les archives micrasiatiques et tous les noms géographiques turcs relevés dans le Fonds Boratav. Cette opération devrait permettre de mettre en relation des noms successifs ou simultanés, turcs et grecs, de même lieux géographiques: ville, village, hameau, lieu-dit, ou relief naturel.

Par la suite nous pourrions envisager également de constituer, selon les mêmes principes, un index de tous les saints, personnages historiques et personnages mythiques présents dans l'un et l'autre corpus. 


\section{XPONIKA}

Ces deux index pourraient, du reste, donner lieu, selon les besoins d'une recherche commune et les moyens alloués, à la saisie des références d'un certain nombre de documents d'archives (travail qui devrait être effectué au niveau de la page ou du groupe de pages constituant la réponse à une des questions de l'interview).

Enfin, et bien que cela ne rentre pas dans le cadre de notre projet initial, il nous paraîtrait du plus haut intérêt d'associer le fonds d'archives musicales à l'élaboration des index.

De même, il nous semblerait hautement souhaitable que les étudiants du département d'ethnologie, se spécialisant dans les domaines concernés, participent aux diverses phases de ce projet.

\section{PROJET DE PUBLICATIONS COMMUNES}

La deuxième partie de notre coopération concerne l'exploitation en commun des deux fonds portant sur les cultures populaires turques et grecques d'Anatolie: le fonds Boratav, déposé au Laboratoire d'ethnologie et de sociologie comparative (UA 140-CNRS), et le fonds du Centre d'Études d'Asie Mineure d'Athènes.

Les matériaux du fonds Boratav proviennent d'une collecte systématique de documents de tradition orale auprès d'informateurs originaires de plusieurs localités de l'Anatolie, recueillis par le professeur P.N. Boratav, présentement Maître de recherche honoraire au CNRS, depuis 1927 à nos jours. Il s'agit d'un ensemble de textes classés par l'auteur en treize grandes rubriques. Ce corpus pourrait facilement se prêter à une comparaison avec les documents du Centre d'Asie Mineure à Athènes; car tant les thèmes que les localités concernées se recoupent. En effet, le fonds Boratav déposé au centre de documentation de l'UA 140-CNRS et qui est en voie d'exploitation informatique porte plutôt sur les populations musulmanes de la Turquie, tandis que les matériaux du centre d'Études d'Asie Mineure d'Athènes concernent les populations chrétiennes (grecs orthodoxes) du pays, et qui se sont installées en Grèce à la suite de l'échange de populations à la fin de la guerre de 1922 - 1923 entre les deux pays.

Comme il est indiqué plus haut (partie 2) la thématique et les catégories communes sont abondantes. A l'issue de notre mission, il nous a semblé souhaitable de commencer ce travail de comparaison en vue de publications communes par une catégorie particulièrement fournie dans les deux cas, celle de la religion populaire et notamment les légendes et les contes 
associés aux lieux et aux personnages qui donnaient lieu à des cultes communs entre chrétiens orthodoxes et musulmans.

On choisira ainsi comme premiers exemples quelques saints du calendrier orthodoxe, connus et vénérés aussi par les populations nonchrétiennes. Le cas le plus exemplaire nous semble être celui d'Agios (Saint) Charalambos, vénéré à la fois par les orthodoxes et par les membres - nombreux en Turquie et dans les Balkans - de l'ordre musulman de tradition chi'ite des Bektashi: le fondateur de cet ordre Haci Bektas Velî est identifié à Agios Charalambos dans ces cultes communs. Par ailleurs, quelques rites populaires (par exemple les rituels et processions pour «faire venir la pluie») seront répertoriés et comparés.

Dans la même démarche comparative, des textes de littérature populaire riches de données relatives à la constitution d'un «modèle héroique» seront également analysés. A cet effet, nous avons choisi dans le fonds du Centre à Athènes deux récits légendaires édités sous forme de brochures de colportage à la fin du Siècle dernier et qui demeurent encore très populaires en milieu turc anatolien. Il s'agit de deux versions de la légende d'Asik Garip ainsi que d'une version du cycle épique de Köröglu («le fils de l'aveugle») publiés en Karamanli (langue des grecs orthodoxes turcophones de l'Anatolie qui, dans sa forme écrite, utilise l'alphabet grec). Ces textes seront comparés aux versions des deux légendes recueillies par Boratav en Anatolie.

Une partie importante de ces travaux comparatifs pourrait aboutir, à très courte échéance, à des publications communes sous l'égide de l'UA 140-CNRS et du Centre d'Athènes. Celles-ci concerneront, pour l'essentiel, les matériaux déjà regroupés dans la rubrique «monuments de la parole» par les chercheurs du Centre d'Études d'Asie Mineure portant sur le folklore (religion populaire et rites calendaires, légendes et mythes, chansons populaires, coutumes et croyances locales). La première de ces publications communes est prévue pour 1988, dans le VIe volume du «Deltion» (Bulletin) du Centre d'Études d'Asie Mineure.

Il convient de signaler enfin une des dimensions déterminantes de cette entreprise d'ethno-histoire comparative menée en coopération. Les échanges de population menées dans les années 1920 entre la Grèce et la Turquie n'eurent pas la même importance pour l'histoire politique, sociale et culturelle des deux pays. La Grèce vit affluer sur son territoire une masse démographique de réfugiés d'Anatolie qui atteignait le cinquième de sa population d'alors. Ces réfugiés venaient naturellement avec leur culture d'origine d'Anatolie et leurs particularismes linguistiques qui influencèrent dans une large mesure la culture nationale grecque, 


\section{XPONIKA}

tout en se fondant en son sein. Ainsi un nombre infime de grecs est en mesure, aujourd'hui, de lire et de comprendre ces documents constitués en «monuments de la parole» par les soins du centre et qui constituent une manière d'archéologie de la culture grecque contemporaine. De son côté, la société turque a évolué à son rythme et avec des apports extérieurs, changeant sa langue et son alphabet, coupant tous les ponts avec le Siècle dernier. La mise en rapport de ces fonds contribuerait ne serait-ce qu'en manière de début, à assurer la complémentarité et la préservation de ces monuments de culture en voie de disparition.

\section{FONDS PERTEV NAILI BORATAV}

Annexe 1. Plan de classement.

$$
\begin{aligned}
& \text { I. } \quad \text { Divers } \\
& \quad-\text { documentation non classée } \\
& \quad-\text { informations } \\
& \text { - recueils de textes } \\
& \text { - cahiers de contes } \\
& \quad-\text { renseignements sur les informateurs }
\end{aligned}
$$

II. Oeuvres des poètes populaires (textes signés)

III. 1. épopées

2. contes (lère série)

IV. 1. contes (2ème série)

2. anecdotes

V. Légendes

VI. 1. proverbes

2. devinettes

3. formules de bénédiction, de malédiction appels à la prière (formules)

VII. Poésie populaire (anonyme)

1. chansons

2. quatrains

3.

4. cantiques (chansons religieuses - anonymes) 
VIII. 1. musique populaire - instruments

2. danse populaire - mélodie

IX. Jeu

1. jeu - sport populaire

2. théâtre populaire

3. autres divertissements populaires

X. Croyances

XI. Cérémonies

1. noces

2. deuil (funérailles)

XII. Traditions matérielles - vie matérielle

1. art populaire

2. artisanat

3. moyens de production et de consommation

4. habitat

5. costumes - ornements

XIII. Institutions religieuses

1. artisanat (religieux)

2. jeunesse

XIV. Sciences populaires

1. magie - talisman

2. médecine populaire - science vétérinaire

3. ethnobotanique

4. astronomie populaire

\section{CENTRE D'ÉTUDES D'ASIE MINEURE}

Annexe 2. Plan de classement des dossiers. Par village.

- Comptes rendus de missions

- Curriculum vitae des informateurs (par un ou plusieurs chercheurs) et appréciations sur leurs qualités d'informateurs

- Matériaux: nombre de documents

\section{Géographie}

A. Toponymie (aspect administratif)

1. nom du village 


\section{XPONIKA}

2. références cartographiques du Centre

3. références à l'administration grecque d'Asie Mineure

4. références à l'administration turque

5. a) références à l'administration ecclésiastique

b) rapports avec les autorités ecclésiastiques

B. Autres informations

1. nombre d'habitants et de familles

2. informations sur les membres connus de la Commission des échanges (1924)

3. références bibliographiques

4. récits des informateurs sur le nombre d'habitants et indications de nom d'autres informateurs possibles

C. Langue

Récits d'informateurs sur le problème de la langue

D. Divers

Récits sur divers aspects tournant autour du nombre d'habitants et autour d'autres informations (description du village par exemple)

E. Géographie physique

1. Situation du village (géographie descriptive)

2. routes d'accès au village

3. montagnes et collines

4. rivières et lacs

5. morphologie du lieu

6. climat

F. Grottes et souterrains

(en rapport avec la situation de la Cappadoce)

G. Lieux-dits

reprise des toponymes par ordre alphabétique avec, pour chacun:

1. le temps pour y parvenir

2. historique du lieu ou étymologie du nom

3. récits sur le folklore, la religion, les rituels, les légendes se rapportant au lieu

4. références à un site antérieur

5. récits se référant à plusieurs toponymes

H. Village
1. quartier
2. rue
3. place, aire de battage
4. puits, citerne, fontaine 
5. maison (description de l'organisation intérieure, de l'agencement des pièces et de leur nom)

6. église, chapelle, sanctuaire

7. école et autres bâtiments

I. Hameaux (proches et lointains)

1. hameaux grecs

2. hameaux turcs

3. autres villages chrétiens devenus turcs

- ancien nom

- histoire d'une islamisation forcée

- réactions populaires à cette islamisation

J. Relations avec les autres villages

1. mariages (cartes de la circulation des femmes)

2. commerce et activités communes (moulin)

II. Vie sociale

A. Vie quotidienne

1. naissance: accouchements (rites, prières, invocations)

2. baptême (moment du...)

3. fiançailles, mariage

- cour: lieux et fêtes, rencontres des jeunes gens; danses; marieuses et "proxénètes"

- mariage: dot et rituel du mariage

4. vie familiale (organisation familiale)

- relations des proches parents entre eux; évitement de l'adresse ou de la parole

- travail des femmes (division sexuelle du travail)

- préparation de la nourriture

- mets traditionnels

- ustensiles de cuisine

- fromage

- pain

- sucré/salé

- répartition des tâches entre hommes et femmes

- recettes de cuisine

- crème fraîche

- gâteaux et mets de Noël

- loisirs et jeux

- jeux

- représentations théâtrales

- jeux à la veille du mariage 


\section{XPONIKA}

- costumes traditionnels

- description coiffure (hommes et femmes)

- description du costume de mariage (hommes et femmes)

- terminologie des pièces du vêtement et croquis

5. mort

- préparation du corps

- description des modes de sépulture

- modes de participation des assistants

- lamentations

- représentations et légendes (de et sur la mort)

- vêtements et coutumes vestimentaires de deuil

- libations sur les tombes

- rassemblement des ossements

- nourriture offerte lors des funérailles

B. Vie religieuse

1. Religion officielle

- les saints

- lieux de culte des différents saints

- pourquoi invoque-t-on tel saint

- miracles dans les lieux de culte

- offrandes de demande

- légendes associées aux cultes des saints

- églises, chapelles, sanctuaires (cf. H6, originaux ici)

- dessin et terminologie

- autels votifs en dehors du village

- îcones (demeurées sur place ou emportées)

- objets sacrés

- clocher

- objets restés sur place ou emportés

- ex-voto

- dans quelles conditions sont-ils réalisés

- pour qui

- en quoi consiste la promesse

- dans quel but

- importance matérielle de l'ex-voto

- rituel du don

- rituel de communion

- fêtes religieuses, jours de repos, prières, carême

- processions, bénédictions des lieux

- fêtes patronales

2. Religion populaire

- cycle des fêtes (cf. le calendrier ecclésiastique et l'almanach ancien) 
- 14 septembre à fin novembrc: récupération de la croix par sainte Hélène

- cycle des 12 jours: Noël, St Basil, Epiphanie, carême, Pâques

- quelques fêtes (St Georges...)

- cycle du mois d'août (Ste Vierge)

- du ler mars au ler mai

- les feux (St Jean...)

- magie

C. Ecoles

(cf I, H.)

D. Sciences populaires

1. Météorologie et astrologie

- météorologie

- noms dans le dialecte des mois et des jours

- informations sur les points cardinaux

- informations sur la météorologie

- dictons sur le temps et expressions populaires

- astrologie

- observation des étoiles

- divination

- heures

- phases de la lune

2. Médecine

- sans religion ni magie

- classement des maladies

- traitement

- avec rituels religieux (associés aux cultes des saints)

- avec rituels religieux et magie (lieux de ces rituels)

- avec magie

- médicaments et prophylaxie

- bains de sable

- cataplasmes

- arbres miraculeux

E. Art populaire et folklore

1. Art populaire

2. Monuments de la parole

- idiomes

- vœux

- chansons

- chants rituels (paroles) 


\section{XPONIKA}

+ Noël

+ St Basil

+ cycle de Pâques

+ cycle du mois d'août

- chants d'amour (parole)

- chants de mariage

- chants de travail

- berceuses

- chants de danse

- légendes chantées

- chants d'adieu (pour ceux qui quittent le pays)

- bibliographie (chants d'après les livres)

- listes des chants dont on ne possède que les premiers mots

- légendes

- contes

3. Danses

\section{F. Economie}

1. Généralités

2. Agriculture

- travaux agricoles

- produits agricoles

- outils agricoles

- mesure (dénomination)

- viticulture

- calendrier agricole

3. Apiculture

4. Élevage

5. Chasse et pêche

6. Commerce

- routes et moyens de transport

- monnaie

7. Professions (magasins et travail à domicile)

- dénombrement et énumération de chacun des deux groupes

- description et dénomination des outils

8. Moulins (blé et huile)

9. Immigration - migration économique

- schéma de vie

- école

- départ vers Istanbul

- travail

- mariage

- retour au village

- métiers

- coutumes 


\section{XPONIKA}

10. Taxes

- terminologie

- coutumes

G. Administration

1. Généralités sur l'administration locale

2. Administration spécifique

H. Droit coutumier

I. Relations Grecs/Turcs

1. Relations de travail

2. Vie sociale

3. Participation des Turcs à la vie religieuse des Grecs (rituels communs)

\section{J. Tribus}

Gens de la montagne, brigands, catégories hors villages

\section{Histoire}

A. Histoire locale

1. Histoire du village et de sa création

2. Monuments historiques

3. Chefs abusifs (turcs)

4. Attaques contre le village par des brigands et persécutions des chrétiens

B. Témoignages sur des événements historiques

1. Affrontements Grecs/Turcs (1821)

2. Guerre turco-russe (1830)

3. Nouveaux Turcs

4. Exode: comment et quand ont-ils quitté les villages

5. L'après exode

- installation en Grèce

- retour en Turquie ultérieur ou contacts - si oui:

- nouveaux noms des villages

- correspondances avec les Turcs

- état des lieux de culte

IV. Bibliographie

A. Sources

1. Registres emportés

2. Manuscrits

B. Ouvrages grecs et étrangers 


\section{XPONIKA}

Références bibliographiques

- Melpo Merlier: Essai d'un tableau du folklore musical grec. Le syllogue pour l'enregistrement des chansons populaires (Athènes, Librairie J.N. Sidéris, 1935), $64 \mathrm{p}$.

- Melpo Merlier: Présentation du Centre d'Etudes d'Asie Mineure. Recherches d'ethnographie (Athènes, 1951), $18 \mathrm{p}$.

- Octave Merlier: Le dernier hellénisme d'Asie Mineure. Introduction à l'exposition du Centre d'Etudes d'Asie Mineure de Melpo Merlier (Athènes, 1974) 51 p., photogr.

MARIA COUROUCLI

MARIE-DOMINIQUE MOUTON 